\title{
ADMINISTRACIÓN DEL CONOCIMIENTO EN LAS EMPRESAS INTERNACIONALES Y SU PRÁCTICA EN EL PERÚ
}

CPC. JULIO PALOMINO SILVA

Docente de la Facultad

\begin{abstract}
RESUMEN
La administración del conocimiento es una infraestructura que comparte información y que puede ser útil para cualquier funcionario en la toma de decisiones. Esta nueva forma de transmitir los conocimientos apareció a finales de los 90; sin embargo, ha tomado auge en los últimos años.

La concentración y almacenamiento de esta información, no consiste solamente en almacenar datos, sino que representa la transmisión de conocimientos, procedimientos o investigaciones a través de una base de datos compartida, apoyada por la tecnología de información para su almacenamiento y acceso, la cual representan una posibilidad de aprovechar el trabajo que ya ha sido desarrollado, ahorrando invertir costos adicionales en un trabajo que ya ha sido realizado.

En este artículo veremos que a través de los años, han existido distintas corrientes gerenciales, las cuales han tenido su época de expansión y en esta época, ha llegado la era de la comunicación y el conocimiento, la llamada "administración del conocimiento" (AC).
\end{abstract}

Palabras clave: Administración del conocimiento (Knowledge management), Center of Business Knowledge, Know how.

\section{INTRODUCCIÓN}

La administración del conocimiento (AC) es una técnica que comenzó a finales de los años 90 y que ha tenido mucho éxito en las organizaciones, se incorporaron algunas tablas que nos muestran los incrementos en las utilidades, asi como los beneficios que se tienen. Las ventajas y beneficios que ha traído a las empresas que han decidido implementar la $\mathrm{AC}$ en su organización. Los puntos importantes que se tienen que tomar en cuenta al momento de realizar el proceso de implementación de la Administración del conocimiento, así como los pilares sobre los cuales descansa; y, por último, las conclusiones y los beneficios que se pueden obtener si se decide implantar está herramienta. 
En la época de globalización, la tecnología de información juega un papel fundamental en todas las organizaciones. Han aparecido varias herramientas para que las organizaciones tengan acceso a la información; sin embargo, esta nueva forma de compartir la información, representa una oportunidad para poder desarrollar un centro de conocimientos.

Eldesarrollo de esta estrategia seha desarrollado apoyándose en la tecnología de información. Simplemente, podriamos decir que cualquier persona en la organización que tenga un conocimiento que aportar a la base de conocimientos, lopuedehacery eseconocimiento después podrá ser utilizado por cualquier otro usuario.

Entre más compleja y más grande sea la organización, mayor va a ser el potencial que se tenga para tener una base de conocimientos muy completa y poderosa y mayor va a ser la ventaja competitiva que va a otorgar.

La administración del conocimiento se ha comenzado a utilizar en las firmas de consultoría y representa una de las mejores oportunidades de incrementar el número de servicios, ganancias y oportunidades de hacer negocios en el mercado del conocimiento.

Si imaginamos todas las posibles áreas en donde esta herramienta puede ser desarrollada, podremos darnos cuenta que los fines pueden ser no sólo lucrativos, sino altruistas. Las áreas en las que esta nueva forma de transmitir el conocimiento son muchas; sin embargo, en las que han tomado gran interés son la educación, investigación y desarrollo científico, así como en las de negocios.

Ya comienzan a haber algunas señales del interés que se tiene para aprovechar este tipo de tecnología en algunos gobiernos que empiezan a visualizar las ventajas y el potencial que esta herramienta representa, por lo que dentro del artículo encontraremos algunos puntos básicos que se deben de tomar en cuenta al momento de implantar un sistema de administración del conocimiento.

\section{OBJETIVOS ESPECÍFICOS}

- Priorizar la adecuada explotación de datos e información para transformarlos en conocimiento y entendimiento, para el mejor desempeño de las funciones en las empresas.

- Documentar el cómo y entender el por qué. Aquí es cuando el conocimiento tácito se vuelve explícito, al entender cómo y por qué un producto o servicio se hizo de mejor forma que otro.

- Las ventajas y beneficios que han traído a las empresas que han decidido implementar la Administración del Conocimiento en su organización además de las Tecnologías de Información.

\section{VENTAJA COMPETITIVA EN LAS ORGA- NIZACIONES}

La administración del conocimiento "AC" es una ventana que se abre a las organizaciones para enfocar sus esfuerzos y considerarla como una nueva herramienta a través de la cual se pueden ofrecer nuevos servicios, siendo un claro ejemplo en el que la tecnología de información se puede alinear a los planes estratégicos de la empresa.

Para poder entender que es la "AC", es necesario tener en claro que significado tiene la palabra conocimiento, para lo cual podemos tomar ladefinición de Leif Edvinsson "capital intelectual es aquel conocimiento que se puede convertir en valor" (Daniel Ochoa, 2000).

Para poder desarrollar un sistema de "AC", es necesario contar con una infraestructura que provea de información correcta y apropiada al personal de una organización, cuando sea 
necesario, sin importar el lugar donde se encuentren, de tal manera que tengan acceso a la información.

De acuerdo a estudios recientes realizados por "Global IT Consulting Report and Kennedy" Research Group", los directores de las firmas de consultoría en Estados Unidos, están enfocando sus esfuerzos en la práctica de la "AC" (Knowledge, 1998).

Los primeros que comenzaron a poner especial atención fueron las llamadas "Big Six" (firmas de auditoría) en los años 90 , aunque en la actualidad, ya casi todas las firmas de auditoría ya tienen este servicio y están obteniendo ganancias considerables derivadas de la incorporación de está práctica.

La mayoría de directores expertos en consultoría especializados en la " $\mathrm{AC}$ " opinan que el suceso del 11 de septiembre de 2001 fue la última razón que confirmó que la $\mathrm{AC}$ era necesaria. El FBI ha tenido muchas críticas acerca de información de inteligencia que se ignoró y que hubieran predicho los ataques terroristas, debido a una falta de comunicación con la CIA (Pamela Babcock, 2004).

Este es un claro ejemplo en el que podemos apreciar la utilidad que puede tener una administración, del conocimiento, y los posibles efectos que podrían tenerse si una organización como el FBI, decide compartir la información y lograr obtener provecho al compartirla, lo cual hubiera podido salvar vidas y evitar la tragedia que todos conocemos.

En el mundo de los negocios, este tipo de situaciones podría traer pérdidas considerables a las organizaciones que decidan no incorporar esta práctica. La revista Fortune menciona que de la lista de las 500 compañías más importantes del mundo, se calcula que las pérdidas ascienden a $\$ 31.5$ billones de dólares, debido a que no se ha incorporado la "AC" en la organización (Pamela, 2004).
Las aplicaciones que se pueden tener son ilimitadas, ya que esta herramienta no sólo es útil en firmas de consultoría. En una organización algunos usos son en el almacenamiento de información como (procesos, políticas, procedimientos, instrucciones, planos, manuales, tips) y cualquier información que se haya utilizado en alguna ocasión para resolver algún problema o contingencia y que va a poder servir y ahorrar tiempo en el futuro.

Imaginemos que entra a una organización en la que el puesto que va a desempeñar es el de jefe de mantenimiento y se encuentra con que la persona que anteriormente estaba en el puesto, tenía 25 años en el puesto y no dejó ninguna información respecto a manuales de procedimientos, instrucciones, planos de las máquinas que se encuentran en la línea de producción, bitácoras de mantenimiento, etc. Obviamente, se sentiría frustrado, sería muy difícil recuperar toda esa información perdida y la compañía tendría que realizar varios desembolsos para lograr reponer toda la información. Sin embargo, si se contara con un sistema de administración del conocimiento, toda está información se tendría en la base de conocimientos y al llegar al primer día a trabajar, se podría continuar con el proceso de mantenimiento, contando con toda la información necesaria para poder llevar a cabo el trabajo.

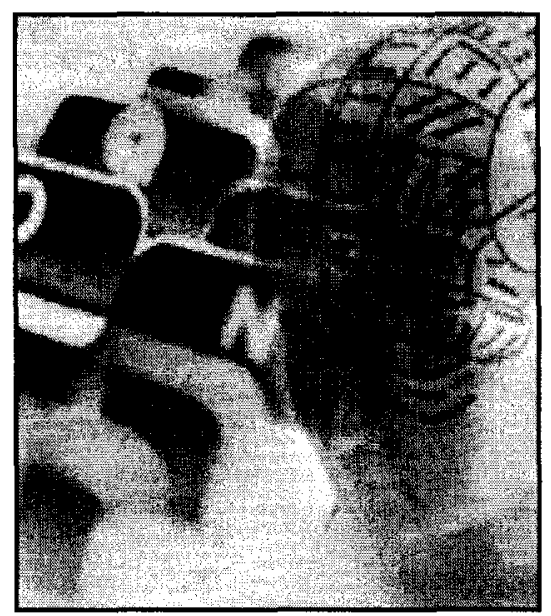




\section{PROCESO DE IMPLEMENTACIÓN}

Para poder analizar el proceso de implantación de un sistema de administración de conocimientos en una empresa específica, nos enfocaremos en algunos pasos que consideramos necesarios que toda empresa consultora debería tener en cuenta:

- Un punto muy importante es que las organizaciones identifiquen en qué áreas (nichos de mercado), se puede maximizar el beneficio; y sólo después de que se hayan identificado las fortalezas, se debe de buscar qué tecnología se debe de utilizar como herramienta e infraestructura para poder compartir la información.

- Definir de qué manera podemos almacenar y distribuir la información, de tal manera que se logra la maximización del uso de dicha información. La firma debe destinar tiempo para analizar. ¿Qué es lo que se ha aprendido con los clientes en el pasado? Discutir: ¿Cómo se puede aprovechar esta información? Y ¿Cómo se puede aplicar dicho conocimiento a otro cliente? Los resultados de estas reuniones deben de ser documentados, incluyendo las agendas, los procesos, yotra información potencial que se identifique que pueda ser de utilidad.

- ¿De qué manera se puede capturar y almacenar la información para aprovechar al máximo los conocimientos que ya se tienen? Es necesario que la firma estandarice alguna manera para capacitar al personal, para que se utilicen las herramientas de manera efectiva y puedan tenerse beneficios de la implementación a corto plazo. Es necesario determinar en qué formatos se van a almacenar la información y considerar medios como audio y video. Es también necesario que el conocimiento almacenado realmente tenga un beneficio; y la manera verificarlo, es probarlo en algún otro cliente. Una vez que se tenga la seguridad de que el resultado fue el esperado, es necesario que esta información sea transmitida al área de entrenamiento, la cual será la responsable de difundirlo. También se puede optar por realizar algún artículo, el cual se pueda hacer público, dependiendo de las expectativas de la firma, o bien ponerlo a disposición de otras firmas que no sean la competencia, de tal manera que lo puedan adquirir de igual manera a como funciona una franquicia, la cual paga regalías por el uso del "Know how".

- ¿Qué herramientas se tienen disponibles o se pueden adquirir para poder entregar y administrar la información a los usuarios de manera más eficiente? La mayor parte de la información que se puede rescatar de una experiencia con un cliente, se puede llevar a papel, por lo que es muy importante que se establezcan procedimientos para almacenar la información, así como un directorio electrónico, en la Intranet en el cual se ponga a disposición de la firma.

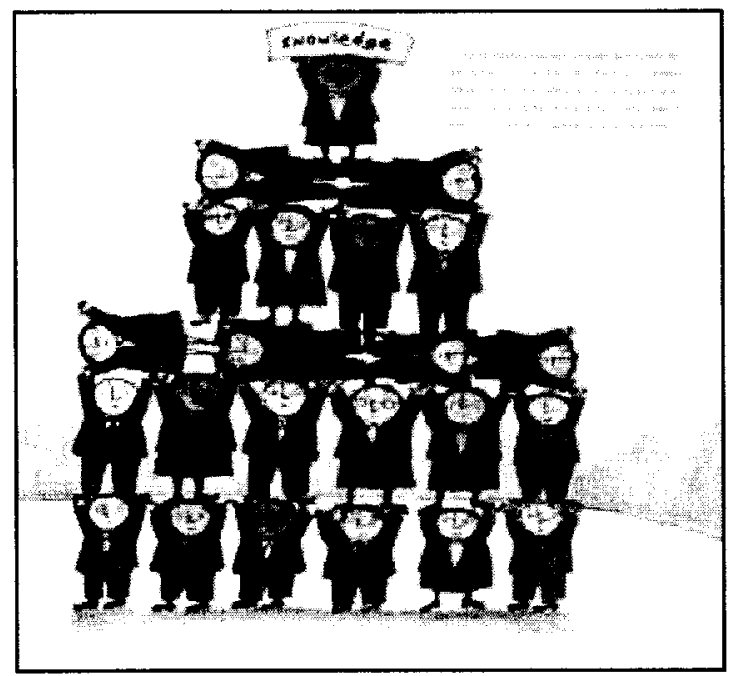


Como es normal en cualquier proceso de implantación de un sistema, existen varios riesgos al fracaso, principalmente cuando el plan implica la incorporación de la tecnología de información. Los principales problemas que se llegan a tener, son cierta resistencia al cambio, la cual se considera como una barrera muy difícil de superar para las personas que forman parte de la organización ya que se les tiene que hacer ver los beneficios que se van a tener al incorporar está nueva tecnología. Otro problema que se puede enfrentar, es lograr vencer la barrera humana que en ocasiones se tiene para compartir información privilegiada, ya que se tiene el miedo de dejar de ser indispensable, al trasmitir la información que poseemos (el know how), y podemos llegar a perder la ventaja competitiva que teníamos cuando éramos los únicos propietarios de la información.

Adicionalmente, existen administradores que no confían en sus empleados, otros tienen miedo de que sus ideas sean ridiculizadas o que simplemente se piensa que este tipo de herramientas implicaría trabajo adicional.

Otro factor que puede afectar el éxito de la mayoría de las iniciativas para implantar la "AC", es debido a que la estrategia no se realiza a largo plazo. La clave no es tanto qué tan rápido termines, sino que tan seguro recorres el camino

Debido a todos estos problemas que se han mencionado, elárea de Recursos Humanos desempeña un papel muy importante en el proceso de implantación de la "AC". Se debería de incentivar en todas las organizaciones algunos instrumentos para motivar a las personas a que utilicen el sistema de administración del conocimiento, a través incentivos económicos (bonos y estímulos económicos), además de un sistemas de promoción, en el cual todos los años antes de realizar las evaluaciones de desempeño, se miden el número de participaciones $\mathrm{y}$ contribuciones al sistema, logrando tener una mayor participación que enriquezca el cerebro de conocimientos de la firma.

Es muy importante para tener éxito que se tenga la habilidad de escuchar las ideas de todos los miembros de la organización, porque no se sabe quién va a ser la persona que realmente aporte una idea que va a servir para resolver o mejorar la situación en cuestión.

\section{PILARES DE LAADMINISTRACIÓN DEL CONOCIMIENTO}

Podemos decir que existen seis pilares importantes en la "AC", El primero es el desarrollar nuevo conocimiento, el segundo es acceder a fuentes externas, el tercero es utilizar el conocimiento que ha sido obtenido, en la toma de decisiones, el cuarto es incorporar el conocimiento, en el producto final que va a ser entregado al cliente, el quinto es tener un listado del conocimiento, en documentos, bases de datos y software, y el último es establecer un proceso para poder medir y evaluar los resultados obtenidos.

En organizaciones importantes como "Xerox", se realizaron algunas prácticas de otorgar incentivos económicos, sin embargo no funcionó, y se buscaron otro tipo de incentivos en base a realmente otorgarle un reconocimiento a los empleados en base a sus aportaciones y resultó ser una medida muy eficaz, ya que los empleados tenían como motivación el ser diferenciados por su participación a la empresa.

Otro ejemplo importante es Motorola y 3M en su división de ciencias, ambos han logrado desarrollar exitosos centros de crecimiento y aprendizaje, los cuales están desarrollados en 
base a la " $A C$ ". En Japón, la firma de detergentes y cosméticos "Kao" se ha dedicado a iniciar una universidad industria, la cual tiene como objetivo ser una fábrica del conocimiento.

Aunque la mayoría de los ejecutivos cree en el concepto de la "AC", al cual consideran es importante para el desarrollo de las empresas, la mayoría no ha iniciado un proceso para implantar esta tecnología a sus organizaciones, ni esperan hacerlo pronto. En una encuesta realizada a 300 directores del área de TI, el 85\% respondió que ellos creen que el esfuerzo por desarrollar la "AC", convertirá a las compañías en entidades más eficientes; sin embargo, solamente el $8 \%$ está actualmente trabajando en esta iniciativa, de los cuales sólo el 7\% está trabajando en estos proyectos, porque los Directores Generales ven a la "AC" como una prioridad.

En un reporte especial publicado por EIU (Economist Inteligence Unit), Estados Unidos, se menciona que alrededor del $62 \%$ de las compañías con más de 10000 empleados tiene sistemas de $A C$; las industrias en las cuales se está teniendo un mayor crecimiento, son las de telecomunicaciones con el $71 \%$, servicios profesionales $58 \%$ y empresas que venden artículos de primera necesidad $57 \%$.

En México, la administración del conocimiento es un tema que ya ha sido valorado por Universidades como el Tec de Monterrey, el cual realizó una alianza con la Universidad de Texas en Austin, para crear la maestría en Ciencias de la Información y Administración del Conocimiento (MIK). El postgrado busca apoyar a todas las organizaciones en la creación de nuevas formas para organizar su información, apoyados en el conocimiento y la tecnología.

Entre los temas que ofrece la maestría, se encuentran: sistemas de valor basados en el conocimiento, administración de capital humano, bases y flujo de información y conocimiento, organización de la información, administración de servicios y organizaciones de información; y recursos de información y servicios de referencia, entre otras.

Sin embargo, es claro que en países como USA, este tema se encuentra ya muy desarrollado, y podríamos decir que está en su época de esplendor y si realizamos una comparación con México, podemos observar que aunque esta herramienta ya se viene utilizando desde finales de los años 90 , nosotros todavía no hemos empezado a desarrollar este tipo de conocimientos. Sin embargo, programas del gobierno federal, como el encabezado por Vicente Fox, E-México le han dado mucha importancia a la transmisión del conocimiento.

\section{CONCLUSIONES}

La administración del conocimiento representa una de las principales herramientas que se tienen en la actualidad para que las organizaciones logren un desarrollo estratégico en el que puedan aprovechar y maximizar la utilización de los conocimientos que se tienen. Es necesario que en nuestro país se desarrolle y se tome cabal importancia de esta herramienta para lograr una mayor eficiencia en la empresa.

Es indiscutible que las organizaciones tienen que alinear la tecnología de información a la estrategia del negocio, por lo que es importante que herramientas como la de administración del conocimiento alcance un desarrollo y madurez a corto plazo.

Esta herramienta apoyada por el uso de la tecnología de información está en su época de auge y podemos apreciar que varias organizaciones están convencidas del beneficio que representa aprovechar conocimientos que ya se tienen y que realmente se pierden y lo más dramático es que en varias ocasiones son investigaciones o procesos que se llevan a cabo 
varias veces y que se tienen que comenzar de cero porque no se cuenta con la documentación apropiada que sirva de referencia.

Las ventajas que ofrece la $\mathrm{AC}$ son reducir costos, acceso a todos los individuos dentro de la organización, mejora continúa, así como un sin numero de beneficios operativos y de negocio que a largo plazo son muchos y en conclusión provoca que el negocio se torne más efectivo $y$ eficiente, ya que las horas de trabajo que se pueden ahorrar son miles.

El mantener información a tu disposición proporciona una ventaja competitiva, ya que se tienen conocimientos al alcance y se puede reaccionar de una manera efectiva al entorno global que hoy en día vivimos.

Es importante mencionar que el proceso para implantar este tipo de sistemas de información r conocimiento no es nada fácil, ya que representa un esfuerzo, en el que todas las áreas de la organización, así como el compromiso de los integrantes para documentar toda la información que pueda llegar a ser compartida en la base de datos, porque el éxito depende en que la información esté documentada en forma adecuada, y pueda utilizarse en el futuro.

\section{REFERENCIAS}

Ochoa, Daniel (03/04/2000). "Capital intelectual / Knowledge management I". El norte de Monterrey [Electronic journal]. http://www. elnorte.com/

"Knowledge management: new directions for IT (and other) consultants.". Journal of Management Consulting Milwaukee [ProQuest]. David Bushko, Michael Raynor

"Ideas into action through knowledge" PWC Trevor Davis and Frank Milton. http:/ / www. pwcglobal.com

Babcock, Pamela (14/05/2004). Shedding light on knowledge management. HRMagazine. www. shrm.ora/hrmagazine.

"Knowledge management:determining what it means in your firm". The CPA Letter New York, oct 2001. [ProQuest]. Roman H. Kepczyk.

Knowledge Management: A new commission for industrial engineers Kevin C. Desouza. Industrial Management. Norcross: jan/feb 2004.

Journal of American Academy of Business (march 2004 ). "Comparison of knowledge management and CMM/CMMI Implementation". Cambridge. Hollywood . 